\title{
The Changes of Polysaccharides in Extracellular Polymeric Substance for Cladophora glomerata under Different Salinity
}

\author{
Yi-Chao Lee, Hwey-Lin Sheu, Shui-Ping Chang*, Chih-Sheng Lee \\ Department of Environmental Engineering \\ Kun Shan University \\ Tainan City, Taiwan (R.O.C.) \\ lyc007@mail.ksu.edu.tw, sheu@mail.ksu.edu.tw, spchang@mail.ksu.edu.tw*, cslee@mail.ksu.edu.tw
}

\begin{abstract}
Cladophora glomera is a genus of widely distributed, global, sessile, large green algae, which is also a euryhaline alga. This study discovered that changes in salinity can induce $C$. glomera to produce massive amounts of extracellular polymeric substance (EPS) when controlling other environmental conditions. If culturing $C$. glomera with salinity greater than a 3.0\% medium for 24 hours, large amounts of EPS will be secreted. And the level of polysaccharides, the primary component of EPS, is slightly decreased in accordance with the increase in the salinity. Therefore, the level of polysaccharides in EPS can be controlled using different salinity in EPS production.
\end{abstract}

Keywords- Cladophora glomerat; extracellular polymeric substance (EPS); salinity; polysaccharide

\section{INTRODUCTION}

C. glomerata (Chlorophyta, Cladophoraceae) is a wide ly distributed, global, sessile, large, filamentous green alg ae commonly found in freshwater areas [1]. It usually is a dominant species and is distributed in waters with diffe rent pollution levels. People often use C. glomerata as an industrial material due to its large growth in the growin $\mathrm{g}$ season, huge biomass, and ease in harvesting. Lately more and more studies have shown that it can also be $\mathrm{u}$ sed as an adsorption material to prevent pollution [2-6], o $\mathrm{r}$ as the adjuvant in medicines [7]. In addition, it is also a unique material after carbonization or graphitization du e to its special fabric structure on the cell walls.

Cladophora is a euryhaline algae the same species can survive in estuaries or lagoons of variable salinity. To adapt to different salinities, Cladophora can change its total lipids as well as the composition of the lipids. As recent research has shown, the EPS of the algae is highly sensitive to salinity. The mucilage layer of C. glomerata is missing when it stays in freshwater areas. Once the salinity of the growing environment is increased, C. glomerata starts to secrete massive EPS so a mucilage layer is formed outside of the cell walls. In recent years, interest in the exploitation of valuable EPSs has been increasing for various industrial applications and renewable resource representing an important class of biotechnological importance [8,9].

Algal is the photosynthetic organism can grow in the different environment and EPSs production which makes algae a perfect candidate for biotechnological exploration.

This study investigated EPS production under different salinity by exploring the features of C. glomerata such as wide distribution, large biomass, and massive EPS induction. This study also investigated the changes of polysaccharides, the primary component of EPS, and hopefully the study results can be used as a reference for valuable EPSs.

\section{METHODS}

\section{A. Algae}

Cladophora is a branched and filamentous algae, the cell walls of which comprise an inner layer of cellulose, a middle layer of pectin, and an outer layer of chitin. In fresh water, lower quantities of EPS can be found on Cladophora. The surface of the algae is rougher, hosting a number of epithets, primarily Bacillariophyta as well as Cladophora, Cyanophyta, and Euglenophyta. C. glomerata usually is the dominant species in its habitat. In Taiwan, the dry weight of algae from freshwater rivers per square meters was up to $117 \mathrm{~g} / \mathrm{m}^{2}$. The $C$. glomerata used in this study was taken from the main river in Taiwan, the upstream of the Chengwun River, and then transferred to aquariums with different salinity for further culturing.

\section{B. Influence of Salinity on C.glomerata EPS}

The sessile $C$. glomerata grew healthily on the carriers (pebbles) and was harvested from the upstream of the Chengwun River (N 23⒉', E 120 $41^{\prime}$ ) in Taiwan. The Chengwun River water above the tidal river reaches was also brought back to the laboratory to prepare a culture medium with different concentrations of salinity, including 8 different salinity levels such as unadjusted (assuming it was $0.0 \%$ ), $0.5 \%$, $1.0 \%$ o, 3.0\%o, 5.0\%o, 10.0\%o, 20.0\%o, and 30.0\%o, so as to culture C. glomerata. Individual aquariums contained 10 liters of river water with different salinity, and the water was circulated using a small motor to create an adequate environment in which to culture C. glomerata. Samples were then taken at $4 \mathrm{~h}, 8 \mathrm{~h}, 12 \mathrm{~h}, 24 \mathrm{~h}, 48 \mathrm{~h}$ and $72 \mathrm{~h}$ to measure variation in mucilage layers and to be extracted EPS for polysaccharides analysis. 


\section{Thickness of Extracellular Mucilage Layer}

To measure the mucilage layer to determine the EPS, we employed the methods used by Tien et al. (2002) [10] and Chiou et al. (2010) [11]. Fig. 1 shows measurements of the mucilage layer of the algae. Algae samples were placed on glass slides with an appropriate quantity of India ink and then observed using an optical microscope. The green regions in the image are the cell walls of Cladophora; the uniform halo on the outside was used to estimate the thickness of the mucilage layer.

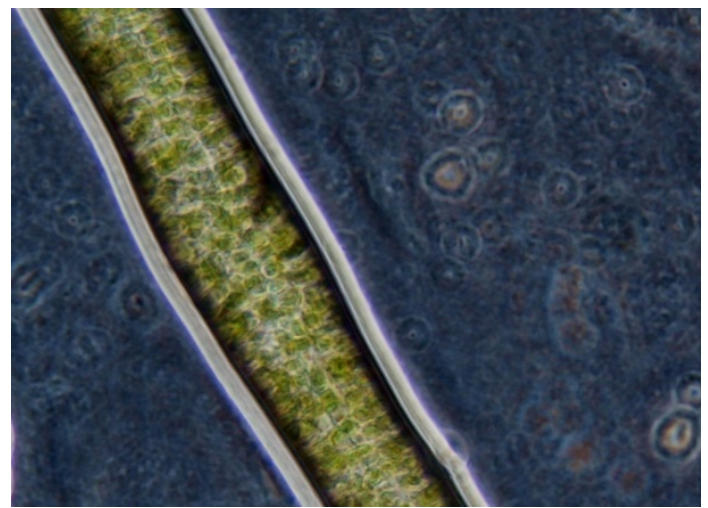

Fig. 1. Illustration of how the measurement of mucilage layer. (the thickness of mucilage layer $=\mathrm{R}-\mathrm{r}$, scale bar $=50 \mu \mathrm{m})$.

\section{EPS Extraction Using Ultrasonication}

There are two types of EPS extraction for algae, physical and chemical extraction methods. Physical extraction is more applicable to large algae. This study used ultrasonication to extract EPS [12, 13]. The algae was harvested from the aquarium, washed twice with tap water, and drained in a strainer at room temperature for $2 \mathrm{~h}$ until it dried naturally. Afterward extraction procedures were started (as indicated in Fig. 2).

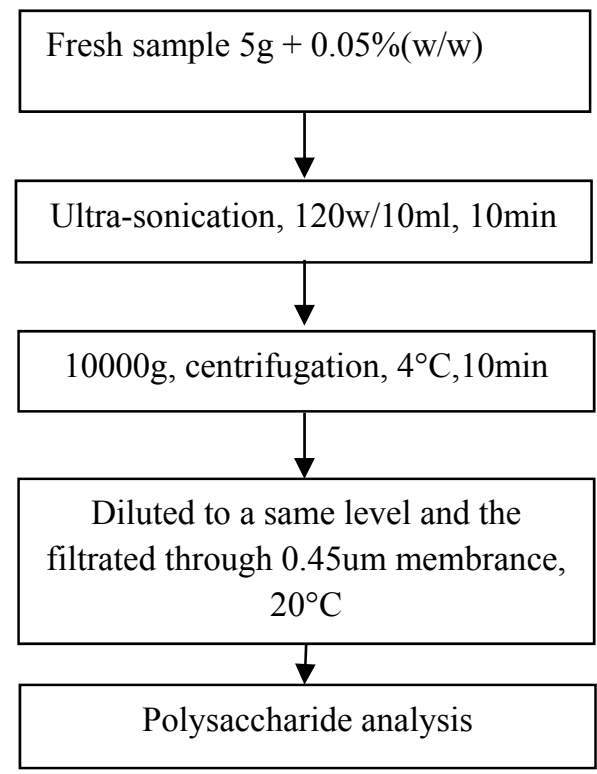

Fig. 2. Procedure for the extraction process of C. glomera EPS for polysaccharide analysis.

\section{E. Analysis of Polysaccharides}

The quantification of crude EPS depends on the thickness of the extracellular mucilage layer. Polysaccharide content was determined by the phenol-sulfuric acid [14], using glucose as a standard. They measured the optical density of hexose at a wavelength of $490 \mathrm{~nm}$ and the optical densities of polysaccharides and uronic acid at a wavelength of $480 \mathrm{~nm}$, and then compared them to the standard curve and calculated the total amount of polysaccharides in the sample.

\section{RESULTS AND DISCUSSION}

\section{A. The Influence of Salinity on the C. glomerata EPS}

To determine the influence of variations in salinity on the EPS and epiphytes of C. glomerata, we conducted experiments using onsite river water and various concentrations of $\mathrm{NaCl}$, $(0.0 \%$ o, $0.5 \%$, $1.0 \%$, $3.0 \%$, $5.0 \%$ o, $10.0 \%$, $20.0 \%$ and $30.0 \%$ o salinity) to cultivate well-grown Cladophora algae samples.

Table I and Fig. 3. demonstrate the changes of $C$. glomerata EPS amounts under culture conditions with different salinity. The results indicated that although EPS secretion was not obvious if the C. glomerata was cultured lower $3.0 \%$ condition, once the culture salinity increased ( $\geqq$ $3.0 \%$ ), EPS secretion also greatly increased accordingly. However, the increment of EPS secretion was not in a portion to the increase in salinity and culture time. In this case, $C$. glomerata, which grows naturally in freshwater areas and thus hardly forms a mucilage layer outside of its cell walls, may start to formulate mucilage layers in accordance with the increase in salinity. To protect itself, the algae could change its physiological conditions to adapt to the environmental changes. But the study results implied that critical salinity for creating significant changes in EPS production was 3.0\%o.

TABLE I. CHANGES OF THE THICKNESS OF MUCILAGE LAYER IN DIFFERENT SALINITY

\begin{tabular}{|c|c|c|c|c|c|c|}
\hline Culinity & $\mathbf{4 h r}$ & $\mathbf{8 h r}$ & $\mathbf{1 2 h r}$ & $\mathbf{2 4 h \mathbf { h }}$ & $\mathbf{4 8 h r}$ & $\mathbf{7 2 h r}$ \\
\hline $1(0.5 \%)$ & 1.35 & 1.35 & 1.35 & 1.40 & 1.45 & 1.47 \\
\hline $2(1.0 \%)$ & 1.62 & 2.50 & 3.72 & 5.45 & 5.90 & 5.97 \\
\hline $3(3.0 \%)$ & 3.67 & 4.40 & 6.73 & 13.28 & 13.80 & 14.17 \\
\hline $4(5.0 \%)$ & 2.70 & 4.70 & 6.12 & 14.23 & 15.07 & 15.57 \\
\hline $5(10.0 \%)$ & 2.75 & 4.07 & 5.30 & 14.50 & 15.70 & 16.75 \\
\hline $6(20.0 \%)$ & 2.75 & 4.32 & 5.92 & 15.33 & 16.42 & 17.25 \\
\hline $7(30.0 \%)$ & 3.68 & 5.18 & 6.93 & 16.23 & 17.55 & 18.17 \\
\hline
\end{tabular}

In this study, we discovered that on C. glomerata, an increase in salinity can accelerate the secretion of EPS, which thickens the extracellular mucilage layer, thereby affecting the attachment of epiphytic algae also. 


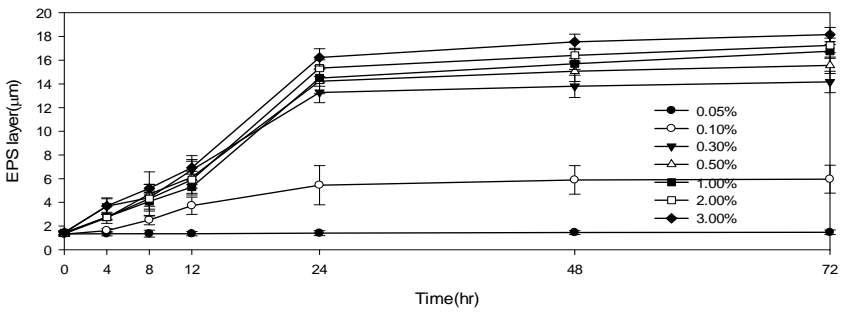

Fig. 3. Changes of the thickness of mucilage.

\section{B. The Influence of Different Salinity on the Level of Polysaccharides in EPS of C. glomerata}

The changes in polysaccharides levels in EPS under different salinity and culture time are shown in Table II and Fig. 4. From the previous results, salinity impacts C. glomerata EPS production. Therefore, changes in salinity may stimulate algae originally grown in freshwater areas into producing a massive amount of EPS. And the total amount of EPS is slightly increased (not significant) in accordance with the increase in salinity and culture time; however, the level of polysaccharides, the primary component of EPS, is contrarily slightly decreased.

TABLE II. POLYSACCHARIDE LEVEL UNDER DIFFERENT SALINITY AND CULTURE TIME

\begin{tabular}{|c|c|c|c|c|c|c|}
\hline Salinity & $4 h r$ & $8 \mathrm{hr}$ & $12 \mathrm{hr}$ & $24 h r$ & $48 \mathrm{hr}$ & $72 \mathrm{hr}$ \\
\hline $1(0 \%)$ & 0.1 & 0.2 & 0.8 & 0.7 & 0.8 & 0.9 \\
\hline $2(0.5 \%)$ & 0.1 & 0.3 & 0.9 & 0.8 & 0.9 & 0.9 \\
\hline $3(1.0 \%)$ & 0.3 & 0.5 & 2.3 & 3.4 & 3.5 & 3.6 \\
\hline $4(3.0 \% 0)$ & 0.4 & 1.0 & 4.3 & 8.6 & 9.0 & 9.2 \\
\hline $5(5.0 \% 0)$ & 0.6 & 1.5 & 3.7 & 8.7 & 9.2 & 9.5 \\
\hline $6(10.0 \% 0)$ & 0.6 & 1.8 & 3.3 & 8.7 & 9.9 & 10.6 \\
\hline $7(20.0 \%)$ & 0.8 & 2.0 & 3.8 & 9.2 & 10.3 & 11.2 \\
\hline $8(30.0 \%)$ & 0.8 & 2.1 & 4.4 & 9.9 & 11.6 & 11.8 \\
\hline
\end{tabular}

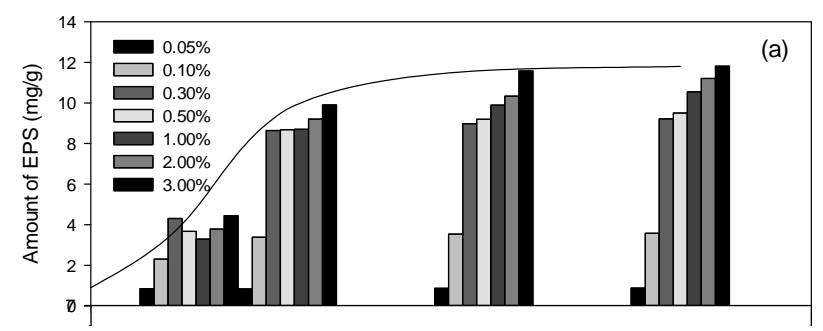

Fig. 4. Variation of polysaccharide level under different salinity and culture time
By exploring the euryhaline feature of C. glomerata, this study discovered that EPS could be massively induced by regulating environmental salinity, and the level of polysaccharides in EPS can also be controlled using the different salinity of habitats when controlling other environmental factors.

\section{SUMMARY}

Cladophora is a genus widely spread all over the world and is also a dominant species, large green algae in estuaries. The C. glomerata used in this study originally grew in freshwater areas, where it hardly secreted EPS. However, EPS could be rapidly secreted due to the changes in salinity in the habitat. Consequently the mucilage layer outside of the cell walls of the algae becomes thicker and thus has a negative impact on the epiphytes of epiphytic algae. This study revealed that EPS secretion was significantly increased when the C. glomerata was cultured in $3.0 \%$ of salinity for 24 hours, but the amount of EPS merely slightly increased in accordance with the increase of salinity and culture time.

The total amount of EPS is slightly increased in accordance with the increase in salinity and culture time; however, the level of polysaccharides is contrarily slightly decreased. This study indicated that EPS production and the level of polysaccharides in EPS can be controlled using changes in salinity. EPSs may allow further exploration of C. glomerata. Studies on the biotechnological importance and ecological significance of the extracellular polymeric substances of $C$. glomerata deserve further attention.

\section{REFERENCES}

[1] W.K. Dodds, and D.A. Gudder, "The ecology of Cladophora", J. Phycol. vol. 28, pp. 415-427, 1992.

[2] L.P. Deng, Y.Y. Su, H. Su, X.T. Wang, and X.B. Zhu, "Sorption and desorption of lead(II) from wastewater by green algae Cladophora fascicularis," J. Hazard. Mater., vol. 143, pp. 220-225, 2007.

[3] N. Oertel, "The applicability of Cladophora glomerata (L.) Kutz in an active biomonitoring technique to monitor heavy metals in the river Danube," Sci. Total Environ., vol. 134, no. S2, pp. 1293-1304, 1993.

[4] A. Singh, S.K. Mehta, and J.P. Gaur, "Removal of heavy metals from aqueous solution by common freshwater filamentous algae," World J. Microb. Biot., vol. 23, pp. 1115-1120, 2007a.

[5] M. Tuzen, A. Sari, "Biosorption of selenium from aqueous solution by green algae (Cladophora hutchinsiae) biomass: equilibrium, thermodynamic and kinetic studies," Chem. Eng. J., vol. 158, pp. 200206, 2010.

[6] Y.C. Lee, and S.P. Chang., "The biosorption of heavy metals from aqueous solution by Spirogyra and Cladophora filamentous macroalgae", Bioresource Technol. vol. 102, pp.5297-5304, 2010.

[7] A. Mihranyan, S.B. Andersson, and R. Ek, "Sorption of nicotine to cellulose powders," Eur. J. Pharm. Sci. vol. 22, pp. 279-286, 2004.

[8] R. Jain, S. Raghukumar, R. Tharanathan, and N.B. Bhosle, "Extracellular polysaccharide production by Thraustochytrid Protists," Marine Biotechnol. vol. 7, pp. 184-192, 2005.

[9] A. Mishra, and B. Jha, "Isolation and characterization of extracellular polymeric substances from micro-algae Dunaliella salina under salt stress," Bioresource Technol. vol. 100, pp. 3382-3386, 2005.

[10] C.J. Tien, V. Krivtsov, E. Levado, D.C. Sigee, and K.N. White, "Occurrence of cell-associated mucilage and soluble extracellular polisaccharides in Rostherne Mere and their possible significance", Hydrobiologia, vol. 485, no. 1-3, pp. 245-252, 2005. 
[11] Y.T. Chiou, M.L. Hsieh, and H.H. Yeh, "Effect of algal extracellular polymer substances on UF membrane fouling" Desalination, vol. 250, pp. 648-652, 2010.

[12] X.M. Han, Z.W. Wang, C.W. Zhu, and Z.C. Wu, "Effect of ultrasonic power density on extracting loosely bound and tightly bound extracellular polymeric substances," Desalination, vol. 329 , no. 15 , pp. $35-40,2013$.

[13] M. Sun, W.W. Li, H.Q. Yu, and H. Harada, "A novel integrated approach to quantitatively evaluate the efficiency of extracellular polymeric substances (EPS) extraction process," Appl. Microbiol. Biotechnol, vol. 96, pp. 1577-1585, 2013.

[14] M. Dubois, K.A. Gilles, J.K. Hamilton, P.A. Rebers, and F. Smith, "Colorimetric method for determination of sugars and related substances," Anal. Chem. vol. 28, no. 3, pp. 350-356, 1956. 